\title{
Experimental constraints on the spin and parity of the $Z(4430)^{+}$
}

K. Chilikin, ${ }^{20}$ R. Mizuk, ${ }^{20,33}$ I. Adachi, ${ }^{12}$ H. Aihara, ${ }^{58}$ K. Arinstein,${ }^{3}$ D. M. Asner ${ }^{44}$ V. Aulchenko, ${ }^{3}$ T. Aushev,${ }^{20}$ T. Aziz, ${ }^{53}$ A. M. Bakich, ${ }^{52}$ A. Bala,${ }^{45}$ V. Bhardwaj,${ }^{36}$ B. Bhuyan, ${ }^{14}$ A. Bondar,${ }^{3}$ G. Bonvicini, ${ }^{64}$ A. Bozek, ${ }^{40}$ M. Bračko, ${ }^{30,21}$ J. Brodzicka, ${ }^{40}$ T. E. Browder, ${ }^{11}$ V. Chekelian, ${ }^{31}$ A. Chen,${ }^{37}$ P. Chen, ${ }^{39}$ B. G. Cheon, ${ }^{10}$ R. Chistov, ${ }^{20}$ I.-S. Cho, ${ }^{66}$ K. Cho, ${ }^{24}$ V. Chobanova, ${ }^{31}$ S.-K. Choi ${ }^{67}$ Y. Choi,${ }^{51}$ D. Cinabro, ${ }^{64}$ J. Dalseno, ${ }^{31,54}$ M. Danilov ${ }^{20,33}$ Z. Doležal, ${ }^{4}$ D. Dutta, ${ }^{14}$ S. Eidelman, ${ }^{3}$ D. Epifanov,${ }^{58}$ H. Farhat,${ }^{64}$ J. E. Fast,${ }^{44}$ T. Ferber, ${ }^{6}$ A. Frey, ${ }^{9}$ V. Gaur ${ }^{53}$ N. Gabyshev,${ }^{3}$ S. Ganguly ${ }^{64}$ R. Gillard ${ }^{64}$ Y. M. Goh, ${ }^{10}$ B. Golob,${ }^{28,}{ }^{21}$ J. Haba, ${ }^{12}$ T. Hara, ${ }^{12}$ K. Hayasaka, ${ }^{35}$ H. Hayashii, ${ }^{36}$ Y. Horii, ${ }^{35}$ Y. Hoshi,${ }^{56}$ W.-S. Hou, ${ }^{39}$ H. J. Hyun, ${ }^{26}$ T. Iijima,${ }^{35,} 34$ A. Ishikawa, ${ }^{57}$ R. Itoh, ${ }^{12}$ Y. Iwasaki, ${ }^{12}$ T. Julius, ${ }^{32}$ D. H. Kah, ${ }^{26}$ J. H. Kang,${ }^{66}$ E. Kato, ${ }^{57}$ T. Kawasaki, ${ }^{42}$ H. Kichimi, ${ }^{12}$ C. Kiesling, ${ }^{31}$ D. Y. Kim, ${ }^{50}$ H. J. Kim,${ }^{26}$ J. B. Kim, ${ }^{25}$ J. H. Kim,${ }^{24}$ K. T. Kim, ${ }^{25}$ Y. J. Kim,${ }^{24}$ K. Kinoshita, ${ }^{5}$ J. Klucar, ${ }^{21}$ B. R. Ko, ${ }^{25}$ P. Kodyš ${ }^{4}$ S. Korpar, ${ }^{30,21}$ P. Križan, ${ }^{28,21}$ P. Krokovny, ${ }^{3}$ T. Kumita, ${ }^{60}$ A. Kuzmin, ${ }^{3}$ Y.-J. Kwon, ${ }^{66}$ J. S. Lange, ${ }^{7}$ S.-H. Lee,${ }^{25}$ J. Li,${ }^{49}$ Y. Li,${ }^{63}$ J. Libby,${ }^{15}$ C. Liu,${ }^{48}$ Y. Liu, ${ }^{5}$ D. Liventsev, ${ }^{12}$ P. Lukin,${ }^{3}$ J. MacNaughton, ${ }^{12}$ D. Matvienko, ${ }^{3}$ K. Miyabayashi, ${ }^{36}$ H. Miyata, ${ }^{42}$ G. B. Mohanty ${ }^{53}$ A. Moll,${ }^{31,54}$ T. Mori ${ }^{34}$ N. Muramatsu ${ }^{47}$ R. Mussa, ${ }^{19}$ E. Nakano, ${ }^{43}$ M. Nakao, ${ }^{12}$ Z. Natkaniec, ${ }^{40}$ M. Nayak, ${ }^{15}$ E. Nedelkovska,${ }^{31}$ C. ${ }^{1 g},{ }^{58}$

N. K. Nisar, ${ }^{53}$ S. Nishida, ${ }^{12}$ O. Nitoh,${ }^{61}$ S. Ogawa, ${ }^{55}$ S. Okuno, ${ }^{22}$ S. L. Olsen,${ }^{49}$ C. Oswald,${ }^{2}$ P. Pakhlov,${ }^{20,33}$ G. Pakhlova, ${ }^{20}$ C. W. Park, ${ }^{51}$ H. Park, ${ }^{26}$ H. K. Park,${ }^{26}$ T. K. Pedlar ${ }^{29}$ R. Pestotnik, ${ }^{21}$ M. Petrič ${ }^{21}$ L. E. Piilonen, ${ }^{63}$

M. Ritter ${ }^{31}$ M. Röhrken, ${ }^{23}$ A. Rostomyan,${ }^{6}$ H. Sahoo, ${ }^{11}$ T. Saito, ${ }^{57}$ K. Sakai, ${ }^{12}$ Y. Sakai, ${ }^{12}$ S. Sandilya,${ }^{53}$ D. Santel,${ }^{5}$ L. Santelj, ${ }^{21}$ T. Sanuki,${ }^{57}$ V. Savinov, ${ }^{46}$ O. Schneider, ${ }^{27}$ G. Schnell,${ }^{1,13}$ C. Schwanda, ${ }^{17}$ D. Semmler, ${ }^{7}$ K. Senyo, ${ }^{65}$ O. Seon, ${ }^{34}$ M. E. Sevior, ${ }^{32}$ M. Shapkin, ${ }^{18}$ C. P. Shen, ${ }^{34}$ T.-A. Shibata, ${ }^{59}$ J.-G. Shiu, ${ }^{39}$ A. Sibidanov, ${ }^{52}$ F. Simon, ${ }^{31,}{ }^{54}$ Y.-S. Sohn, ${ }^{66}$ A. Sokolov ${ }^{18}$ E. Solovieva, ${ }^{20}$ M. Starič ${ }^{21}$ M. Steder,${ }^{6}$ M. Sumihama, ${ }^{8}$ T. Sumiyoshi, ${ }^{60}$

U. Tamponi, ${ }^{19,62}$ K. Tanida, ${ }^{49}$ G. Tatishvili, ${ }^{44}$ Y. Teramoto,${ }^{43}$ K. Trabelsi, ${ }^{12}$ M. Uchida ${ }^{59}$ S. Uehara, ${ }^{12}$

Y. Unno, ${ }^{10}$ S. Uno, ${ }^{12}$ P. Urquijo, ${ }^{2}$ Y. Usov ${ }^{3}$ S. E. Vahsen, ${ }^{11}$ C. Van Hulse,${ }^{1}$ P. Vanhoefer,${ }^{31}$ G. Varner, ${ }^{11}$

K. E. Varvell,${ }^{52}$ A. Vinokurova,${ }^{3}$ V. Vorobyev,${ }^{3}$ M. N. Wagner,${ }^{7}$ C. H. Wang, ${ }^{38}$ M.-Z. Wang, ${ }^{39}$ P. Wang, ${ }^{16}$

X. L. Wang ${ }^{63}$ M. Watanabe, ${ }^{42}$ Y. Watanabe,${ }^{22}$ K. M. Williams, ${ }^{63}$ E. Won,${ }^{25}$ B. D. Yabsley,${ }^{52}$ H. Yamamoto,${ }^{57}$

Y. Yamashita, ${ }^{41}$ S. Yashchenko, ${ }^{6}$ Y. Yook ${ }^{66}$ Y. Yusa, ${ }^{42}$ Z. P. Zhang, ${ }^{48}$ V. Zhilich, ${ }^{3}$ V. Zhulanov, ${ }^{3}$ and A. Zupanc ${ }^{23}$

(The Belle Collaboration)

${ }^{1}$ University of the Basque Country UPV/EHU, 48080 Bilbao

${ }^{2}$ University of Bonn, 53115 Bonn

${ }^{3}$ Budker Institute of Nuclear Physics SB RAS and Novosibirsk State University, Novosibirsk 630090

${ }^{4}$ Faculty of Mathematics and Physics, Charles University, 12116 Prague

${ }^{5}$ University of Cincinnati, Cincinnati, Ohio 45221

${ }^{6}$ Deutsches Elektronen-Synchrotron, 22607 Hamburg

${ }^{7}$ Justus-Liebig-Universität Gießen, 35392 Gießen

${ }^{8}$ Gifu University, Gifu 501-1193

${ }^{9}$ II. Physikalisches Institut, Georg-August-Universität Göttingen, 37073 Göttingen

${ }^{10}$ Hanyang University, Seoul 133-791

${ }^{11}$ University of Hawaii, Honolulu, Hawaii 96822

${ }^{12}$ High Energy Accelerator Research Organization (KEK), Tsukuba 305-0801

${ }^{13}$ Ikerbasque, 48011 Bilbao

${ }^{14}$ Indian Institute of Technology Guwahati, Assam 781039

${ }^{15}$ Indian Institute of Technology Madras, Chennai 600036

${ }^{16}$ Institute of High Energy Physics, Chinese Academy of Sciences, Beijing 100049

${ }^{17}$ Institute of High Energy Physics, Vienna 1050

${ }^{18}$ Institute for High Energy Physics, Protvino 142281

${ }^{19}$ INFN - Sezione di Torino, 10125 Torino

${ }^{20}$ Institute for Theoretical and Experimental Physics, Moscow 117218

${ }^{21} \mathrm{~J}$. Stefan Institute, 1000 Ljubljana

${ }^{22}$ Kanagawa University, Yokohama 221-8686

${ }^{23}$ Institut für Experimentelle Kernphysik, Karlsruher Institut für Technologie, 76131 Karlsruhe

${ }^{24}$ Korea Institute of Science and Technology Information, Daejeon 305-806

${ }^{25}$ Korea University, Seoul 136-713

${ }^{26}$ Kyungpook National University, Daegu 702-701

${ }^{27}$ École Polytechnique Fédérale de Lausanne (EPFL), Lausanne 1015

${ }^{28}$ Faculty of Mathematics and Physics, University of Ljubljana, 1000 Ljubljana

${ }^{29}$ Luther College, Decorah, Iowa 52101

${ }^{30}$ University of Maribor, 2000 Maribor

${ }^{31}$ Max-Planck-Institut für Physik, 80805 München 


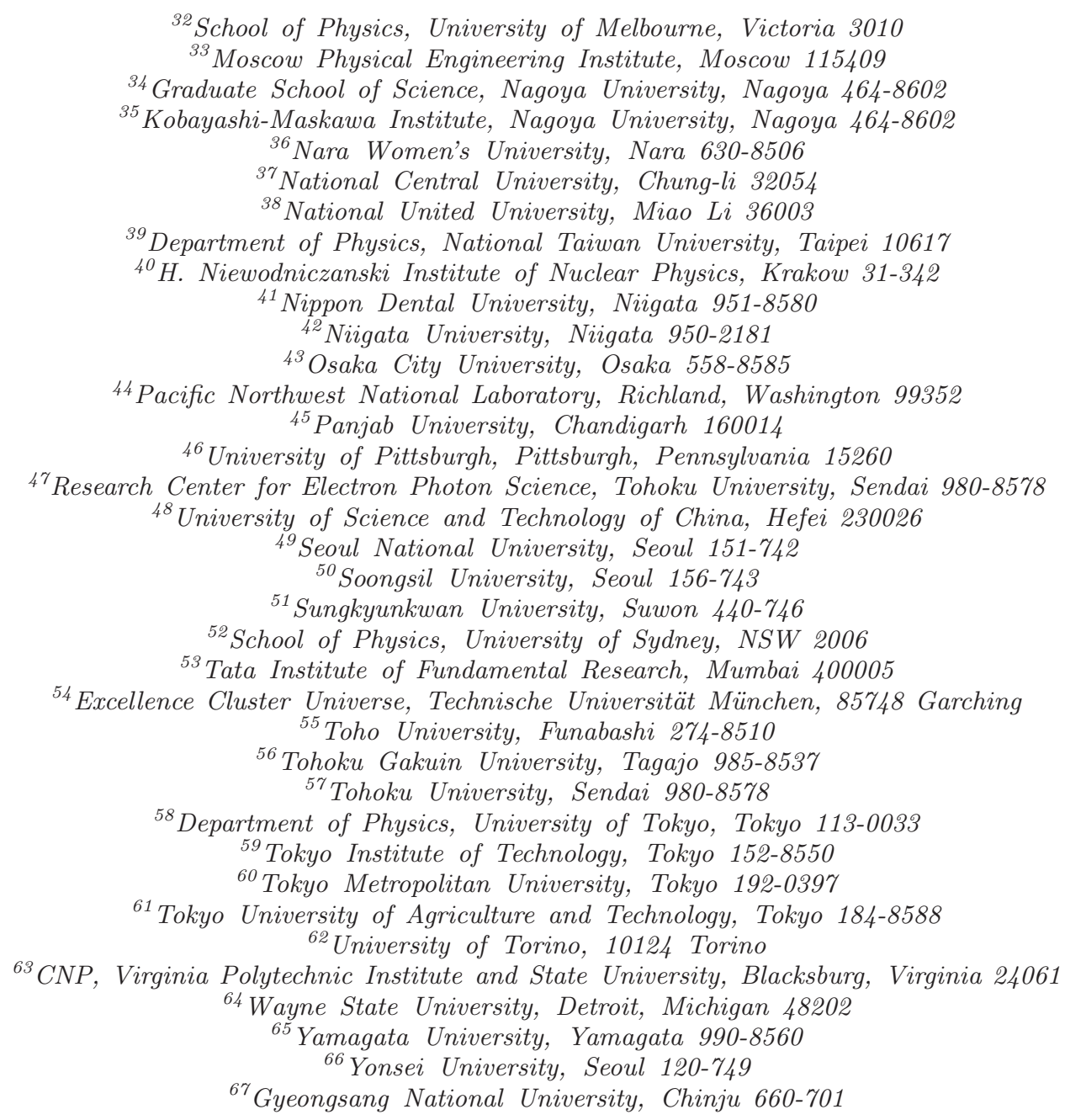

We perform a full amplitude analysis of $B^{0} \rightarrow \psi^{\prime} K^{+} \pi^{-}$decays, with $\psi^{\prime} \rightarrow \mu^{+} \mu^{-}$or $e^{+} e^{-}$, to constrain the spin and parity of the $Z(4430)^{-}$. The $J^{P}=1^{+}$hypothesis is favored over the $0^{-}, 1^{-}$, $2^{-}$and $2^{+}$hypotheses at the levels of $3.4 \sigma, 3.7 \sigma, 4.7 \sigma$ and $5.1 \sigma$, respectively. The analysis is based on a $711 \mathrm{fb}^{-1}$ data sample that contains $772 \times 10^{6} B \bar{B}$ pairs collected at the $\Upsilon(4 S)$ resonance by the Belle detector at the asymmetric-energy $e^{+} e^{-}$collider KEKB.

PACS numbers: 14.40.Nd, 14.40.Rt, 13.25.-k

\section{INTRODUCTION}

Recently, a number of new states containing a $c \bar{c}$ quark pair have been observed, many of which are not well described by the quark model [1, 2]. Among these states are charged charmonium-like state candidates; their minimal quark content is necessarily exotic: $|c \bar{c} u \bar{d}\rangle$. The Belle Collaboration observed a resonance-like structure, the $Z(4430)^{+}$, in the $\psi^{\prime} \pi^{+}$invariant mass spectrum in $\bar{B}^{0} \rightarrow \psi^{\prime} K^{-} \pi^{+}$decays [3, 4]. Two resonance-like structures, the $Z(4050)^{+}$and $Z(4250)^{+}$, were observed in the $\chi_{c 1} \pi^{+}$invariant mass spectrum in $\bar{B}^{0} \rightarrow \chi_{c 1} K^{-} \pi^{+}$decays [5]. The BaBar collaboration searched for these states in $\bar{B}^{0} \rightarrow \psi^{\prime} K^{-} \pi^{+}$and $\bar{B}^{0} \rightarrow J / \psi K^{-} \pi^{+}$decays [6] and in $\bar{B}^{0} \rightarrow \chi_{c 1} K^{-} \pi^{+}$decay [7] but did not confirm them. The BESIII and Belle Collaborations also ob- served the $Z(3900)^{ \pm}$in the $J / \psi \pi^{ \pm}$invariant mass spectrum in $Y(4260) \rightarrow J / \psi \pi^{+} \pi^{-}$decays [8, 9].

The results described in Ref. 4] are based on a twodimensional Dalitz analysis. Here we present the results of a full amplitude analysis of the same decay $B^{0} \rightarrow \psi^{\prime} K^{+} \pi^{-}$, with $\psi^{\prime} \rightarrow \mu^{+} \mu^{-}$or $e^{+} e^{-}$; the decay channel $\psi^{\prime} \rightarrow J / \psi \pi^{+} \pi^{-}$is omitted due to higher multiplicity of the final state. The full amplitude analysis is more sensitive to the $Z(4430)^{-}$quantum numbers than a Dalitz analysis because there is no information loss due to integration over angular variables. The analysis is performed using a $711 \mathrm{fb}^{-1}$ data sample collected by the Belle detector at the asymmetric-energy $e^{+} e^{-}$collider KEKB [10]. The data sample was collected at the $\Upsilon(4 S)$ resonance and contains $772 \times 10^{6} B \bar{B}$ pairs. 


\section{THE BELLE DETECTOR}

The Belle detector is a large-solid-angle magnetic spectrometer that consists of a silicon vertex detector (SVD), a 50-layer central drift chamber (CDC), an array of aerogel threshold Cherenkov counters (ACC), a barrel-like arrangement of time-of-flight scintillation counters (TOF), and an electromagnetic calorimeter comprised of $\mathrm{CsI}(\mathrm{Tl})$ crystals (ECL) located inside a superconducting solenoid coil that provides a $1.5 \mathrm{~T}$ magnetic field. An iron flux return located outside of the coil is instrumented to detect $K_{L}^{0}$ mesons and to identify muons (KLM). The detector is described in detail elsewhere [11]. Two inner detector configurations were used. A $2.0 \mathrm{~cm}$ beampipe and a 3 layer silicon vertex detector were used for the first sample of $140 \mathrm{fb}^{-1}$, while a $1.5 \mathrm{~cm}$ beampipe, a 4-layer silicon detector and a small-cell inner drift chamber were used to record the remaining $571 \mathrm{fb}^{-1}[12$ ].

We use a GEANT-based Monte Carlo (MC) simulation [13] to model the response of the detector, identify potential backgrounds and determine the acceptance. The MC simulation includes run-dependent detector performance variations and background conditions. Signal MC events are generated with Evtgen [14] in proportion to the relative luminosities of the different running periods.

\section{EVENT SELECTION}

We select events of the type $B^{0} \rightarrow \psi^{\prime} K^{+} \pi^{-}$(inclusion of charge-conjugate modes being implied), where the $\psi^{\prime}$ meson is reconstructed via its $e^{+} e^{-}$and $\mu^{+} \mu^{-}$decay channels.

All tracks are required to originate from the interaction point region, $d r<0.2 \mathrm{~cm}$ and $|d z|<2 \mathrm{~cm}$, where $d r$ and $d z$ are the cylindrical coordinates of the point of closest approach of the track to the beam axis. The $z$ axis of the reference frame coincides with the positron beam axis; its origin is the interaction point. Charged $\pi$ and $K$ mesons are identified using likelihood ratios $R_{\pi / K}=\mathcal{L}_{\pi} /\left(\mathcal{L}_{\pi}+\mathcal{L}_{K}\right)$ and $R_{K / \pi}=\mathcal{L}_{K} /\left(\mathcal{L}_{\pi}+\mathcal{L}_{K}\right)$, where $\mathcal{L}_{\pi}$ and $\mathcal{L}_{K}$ are the likelihoods for $\pi$ and $K$, respectively, that are calculated from the combined timeof-flight information from the TOF, the number of photoelectrons from the ACC and $d E / d x$ measurements in the CDC. We require $R_{\pi / K}>0.6$ for $\pi$ candidates and $R_{K / \pi}>0.6$ for $K$ candidates. The $K$ identification efficiency is typically $90 \%$ and the misidentification probability is about $10 \%$. Muons are identified by their range and transverse scattering in the KLM. Electrons are identified by the presence of a matching electromagnetic shower in the ECL. An electron veto is imposed on $\pi$ and $K$ candidates.

For $\psi^{\prime} \rightarrow e^{+} e^{-}$candidates, we include photons that have energies greater than $30 \mathrm{MeV}$ and are within 50 mrad of the lepton direction in the calculation of the $\psi^{\prime}$ invariant mass. We require $\left|M\left(\ell^{+} \ell^{-}\right)-m_{\psi^{\prime}}\right|<$
$60 \mathrm{MeV} / c^{2}$, where $\ell$ is either $\mu$ or $e$. We perform a massconstrained fit to the $\psi^{\prime}$ candidates.

The beam-energy-constrained mass of the $B$ meson is defined as $M_{\mathrm{bc}}=\sqrt{E_{\mathrm{beam}}-\left(\sum_{i} \vec{p}_{i}\right)^{2}}$, where $E_{\mathrm{beam}}$ is the beam energy in the center-of-mass frame and $\vec{p}_{i}$ are momenta of decay products in the same frame. We require $\left|M_{\mathrm{bc}}-m_{B}\right|<7 \mathrm{MeV} / c^{2}$, where $m_{B}$ is the $B^{0}$ mass [15]. A mass-constrained fit is applied to the $B$ meson candidates.

\section{EVENT DISTRIBUTIONS AND SIGNAL YIELD}

The difference between the reconstructed energy and the beam energy $\Delta E=\sum_{i} E_{i}-E_{\text {beam, }}$, where $E_{i}$ are energies of the $B^{0}$ decay products, is used to identify the signal. The signal region is defined as $|\Delta E|<15 \mathrm{MeV}$, and the sidebands are defined as $30 \mathrm{MeV}<|\Delta E|<$ $45 \mathrm{MeV}$. The $\Delta E$ distribution is shown in Fig. 1]

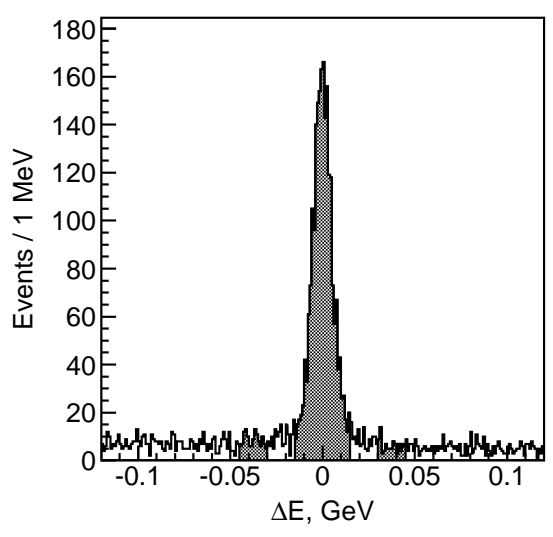

FIG. 1: The $\Delta E$ distribution; the signal and sideband regions are hatched.

To determine the signal and background event yields, we perform a binned maximum likelihood fit of the $\Delta E$ distribution. It is fitted to the sum of two Gaussian functions to represent the signal and a second-order polynomial for the background; all parameters are free. The total number of events in the signal region is 2181; the number of signal events in the signal region is determined to be $2010 \pm 50 \pm 40$ (here and elsewhere in the paper, the first uncertainty is statistical and the second is systematic). Systematic errors are estimated by changing the $\Delta E$ fit interval and the order of the polynomial. We find multiple candidates in $1.4 \%$ of events; no best candidate selection is applied.

The Dalitz distribution of $M_{K^{-} \pi^{+}}^{2}$ vs $M_{\psi^{\prime} \pi^{+}}^{2}$ for the signal region is shown in Fig. 2(a). The vertical band due to production of the intermediate $K^{*}(892)$ resonance is clearly visible. The Dalitz distribution in Fig. 2(b) for the sidebands is featureless. 

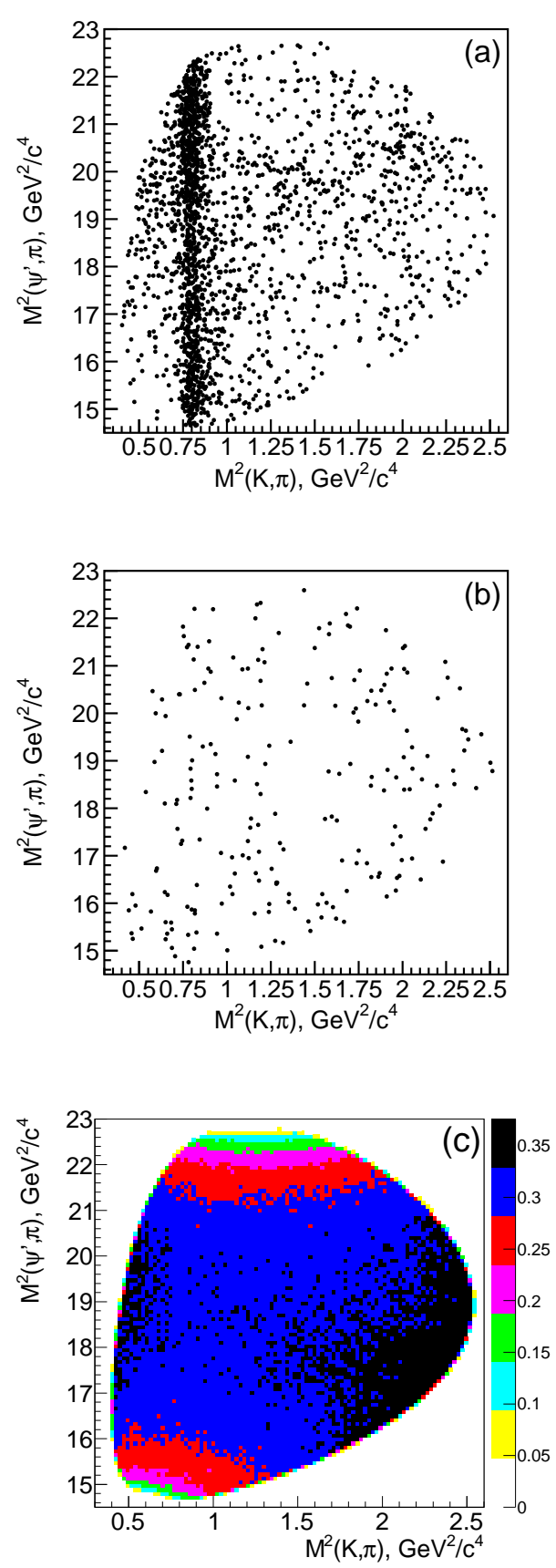

FIG. 2: The Dalitz plots of the signal region (a), sidebands (b) and efficiency (c).

To calculate the reconstruction efficiency, we generate $\mathrm{MC}$ events for $B^{0} \rightarrow \psi^{\prime}\left(\rightarrow \ell^{+} \ell^{-}\right) K^{+} \pi^{-}$with a uniform phase space distribution. The efficiency is corrected for the difference between the particle identification efficiency in data and MC, which is obtained from a $D^{*+} \rightarrow D^{0}\left(\rightarrow K^{-} \pi^{+}\right) \pi^{+}$control sample for $K$ and $\pi$ and a sample of $\gamma \gamma \rightarrow \ell^{+} \ell^{-}$for $\mu$ and $e$.

The efficiency as a function of the Dalitz variables is shown in Fig. 2(c). The efficiency drops in the lower left corner due to slow pions and in the upper corner due to slow kaons; elsewhere it is almost flat. The efficiency as a function of the angular variables is shown in Fig. 3. $\theta_{\psi^{\prime}}$ is the $\psi^{\prime}$ helicity angle [the angle between the momenta of the $\left(K^{+}, \pi^{-}\right)$system and the $\mu^{-}$in the $\psi^{\prime}$ rest frame] and $\varphi$ is the angle between the planes defined by the $\left(\ell^{+}, \ell^{-}\right)$and $\left(K^{+}, \pi^{-}\right)$momenta in the $B^{0}$ rest frame. The efficiency variation in these distributions is at the $10 \%$ level.
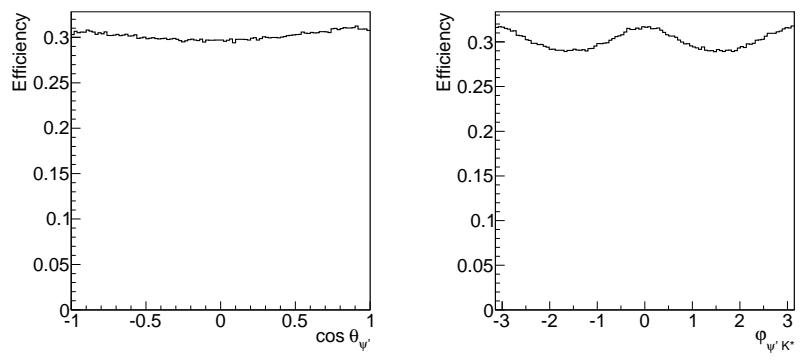

FIG. 3: Efficiency as a function of angular variables.

\section{AMPLITUDE ANALYSIS FORMALISM}

The amplitude of the decay $B^{0} \rightarrow \psi^{\prime} K^{+} \pi^{-}$is represented by the sum of Breit-Wigner contributions for several intermediate two-body states. Our default fit model includes all known $K^{+} \pi^{-}$resonances below the kinematic boundary $\left(1593 \mathrm{MeV} / c^{2}\right)\left[K^{*}{ }_{0}(800), K^{*}(892)\right.$, $\left.K^{*}(1410), K^{*}{ }_{0}(1430), K^{*}{ }_{2}(1430)\right]$, the first resonance above the boundary $\left[K^{*}(1680)\right]$, and an exotic $\psi^{\prime} \pi^{+}$resonance.

The amplitude is calculated in a four-dimensional parameter space, defined by

$$
\Phi=\left(M_{K^{-} \pi^{+}}^{2}, M_{\psi^{\prime} \pi^{+}}^{2}, \theta_{\psi^{\prime}}, \varphi\right) .
$$

The angle-independent part of the amplitude for the decay $B^{0} \rightarrow \psi^{\prime} K^{+} \pi^{-}$via a two-body intermediate resonance $R$ (where $R$ denotes either a $K^{+} \pi^{-}$or $\psi^{\prime} \pi^{-}$resonance) is given by

$$
A^{R}\left(M_{R}^{2}\right)=\frac{F_{B}^{\left(L_{B}\right)} F_{R}^{\left(L_{R}\right)}\left(\frac{p_{B}}{m_{B}}\right)^{L_{B}}\left(\frac{p_{R}}{M_{R}}\right)^{L_{R}}}{m_{R}^{2}-M_{R}^{2}-i m_{R} \Gamma\left(M_{R}\right)},
$$

where $M_{R}$ is the invariant mass of two daughters of the $R$ resonance; $F_{B}^{\left(L_{B}\right)}$ and $F_{R}^{\left(L_{R}\right)}$ are the $B^{0}$ meson and $R$ resonance decay form factors (the superscript denoting the orbital angular momentum of the decay); $\left(p_{B} / m_{B}\right)^{L_{B}} \cdot\left(p_{R} / M_{R}\right)^{L_{R}}$ is related to the momentum dependence of the wave function, with $p_{B}\left(p_{R}\right)$ being the $B^{0}$ meson ( $R$ resonance) daughter's momentum in the $B(R)$ rest frame; $m_{B}$ is the $B^{0}$ meson mass; $m_{R}$ is the 
mass and $\Gamma\left(M_{R}\right)$ is the energy-dependent width of the $R$ resonance. The formula (2) is the same as in the previous Belle analyses [4, 5]. The angle-independent part of the nonresonant amplitude is given by Eq. (2) without the denominator.

We use the Blatt-Weisskopf form factors [16]:

$$
\begin{aligned}
& F^{(0)}=1, \\
& F^{(1)}=\sqrt{\frac{1+z_{0}}{1+z}}, \\
& F^{(2)}=\sqrt{\frac{z_{0}^{2}+3 z_{0}+9}{z^{2}+3 z+9}}, \\
& F^{(3)}=\sqrt{\frac{z_{0}^{3}+6 z_{0}^{2}+45 z_{0}+225}{z^{3}+6 z^{2}+45 z+225}},
\end{aligned}
$$

where $z=r^{2} p_{R}^{2}$ ( $r$ being the hadron scale) and $z_{0}=$ $r^{2} p_{R 0}^{2}$, where $p_{R 0}$ is the $R$ resonance daughter's momentum calculated for the pole mass of the $R$ resonance. For $K^{*}$ resonances with nonzero spin $J$, the $B$ decay orbital angular momentum $L_{B}$ can have the values $J-1, J$ and $J+1$. We take the lowest allowed $L_{B}$ as the default value and consider the other possibilities in the systematic uncertainty. The energy-dependent width is parametrized as

$$
\Gamma\left(M_{R}\right)=\Gamma_{0} \cdot\left(p_{R} / p_{R 0}\right)^{2 L_{R}+1} \cdot\left(m_{R} / M_{R}\right) \cdot F_{R}^{2} .
$$

The angle-dependent part of the amplitude is obtained using the helicity formalism. The amplitude of the decay $B^{0} \rightarrow \psi^{\prime}\left(\rightarrow \ell^{+} \ell^{-}\right) K^{*}\left(\rightarrow K^{+} \pi^{-}\right)$for one $K^{*}$ resonance is

$$
\begin{aligned}
A_{\lambda \xi}^{K^{*}}(\Phi)= & H_{\lambda}^{K^{*}} A^{K^{*}}\left(M_{K^{-} \pi^{+}}^{2}\right) d_{\lambda 0}^{J\left(K^{*}\right)}\left(\theta_{K^{*}}\right) \\
& \times e^{i \lambda \varphi} d_{\lambda \xi}^{1}\left(\theta_{\psi^{\prime}}\right),
\end{aligned}
$$

where $\lambda$ is the helicity of the $\psi^{\prime}$ (the quantization axis being parallel to the $K^{*}$ momentum in the $\psi^{\prime}$ rest frame); $\xi$ is the helicity of the lepton pair; $H_{\lambda}^{R}$ is the helicity amplitude for the decay via the intermediate resonance $R ; d_{\lambda 0}^{J\left(K^{*}\right)}$ and $d_{\lambda \xi}^{1}$ are Wigner $d$ functions; $J\left(K^{*}\right)$ is the spin of the $K^{*}$ resonance and $\theta_{K^{*}}$ is the $K^{*}$ helicity angle (the angle between the $\psi^{\prime}$ and $\pi^{-}$momenta in the $K^{*}$ rest frame). For $K^{*}$ resonances with spin 0 , only $\lambda=0$ is allowed. The angle-dependent part of the nonresonant amplitude is given by Eq. (5) with relative angular momentum between the $K^{+}$and $\pi^{-}$instead of $J\left(K^{*}\right)$.

For the decay $B^{0} \rightarrow K^{+} Z^{-}\left(\rightarrow \psi^{\prime}\left(\rightarrow \ell^{+} \ell^{-}\right) \pi^{-}\right)$, the amplitude is

$$
\begin{aligned}
A_{\lambda^{\prime} \xi}^{Z^{-}}(\Phi)= & H_{\lambda^{\prime}}^{Z^{-}} A^{Z^{-}}\left(M_{\psi^{\prime} \pi^{+}}^{2}\right) d_{0 \lambda^{\prime}}^{J\left(Z^{-}\right)}\left(\theta_{Z^{-}}\right) \\
& \times e^{i \lambda^{\prime} \tilde{\varphi}} d_{\lambda^{\prime} \xi}^{1}\left(\tilde{\theta}_{\psi^{\prime}}\right) e^{i \xi \alpha}
\end{aligned}
$$

where $\lambda^{\prime}$ is the helicity of the $\psi^{\prime}$ (the quantization axis being parallel to the $\pi^{-}$momentum in the $\psi^{\prime}$ rest frame); $\theta_{Z^{-}}$is the $Z^{-}$helicity angle (the angle between the $K^{+}$ and $\pi^{-}$momenta in the $Z^{-}$rest frame); $\tilde{\varphi}$ is the angle between the planes defined by the $\left(\ell^{+}, \pi^{-}\right)$and $\left(K^{+}, \pi^{-}\right)$ momenta in the $\psi^{\prime}$ rest frame; $\tilde{\theta}_{\psi^{\prime}}$ is the $\psi^{\prime}$ helicity angle (the angle between the $\pi^{-}$and $\mu^{-}$momenta in the $\psi^{\prime}$ rest frame); $\alpha$ is the angle between the planes defined by the $\left(\ell^{+}, \pi^{-}\right)$and $\left(\ell^{+}, K^{*}\right)$ momenta in the $\psi^{\prime}$ rest frame. If the spin of the $Z^{-}$equals 0 , only $\lambda^{\prime}=0$ is allowed. The amplitudes in Eq. (6) for different $\lambda^{\prime}$ values are related by parity conservation:

$$
H_{\lambda^{\prime}}^{Z^{-}}=-P\left(Z^{-}\right)(-1)^{J\left(Z^{-}\right)} H_{-\lambda^{\prime}}^{Z^{-}}
$$

The resulting expression for the signal density function

$$
S(\Phi)=\sum_{\xi=1,-1}\left|\sum_{K^{*}} \sum_{\lambda=-1,0,1} A_{\lambda \xi}^{K^{*}}+\sum_{\lambda^{\prime}=-1,0,1} A_{\lambda^{\prime} \xi}^{Z^{-}}\right|^{2} .
$$

A detailed description of the derivation of the amplitude is given in the Appendix.

We perform an unbinned maximum likelihood fit in the four-dimensional space $\Phi$. The construction of the likelihood function follows Ref. [17]. The function to be minimized is

$$
F=-2 \sum_{i} \ln \left((1-b) \frac{S\left(\Phi_{i}\right)}{\sum_{j} S\left(\Phi_{j}\right)}+b \frac{B\left(\Phi_{i}\right)}{\sum_{j} B\left(\Phi_{j}\right)}\right)
$$

where $b$ is the fraction of the background events and $B(\Phi)$ is the background density in the signal region. The sum $\sum_{i}$ runs over data events; the sum $\sum_{j}$ runs over MC events generated uniformly over the phase space and reconstructed using the same selection requirements as in data. This procedure takes into account the nonuniformity of the reconstruction efficiency but requires a parametrization of the background shape.

As there is sensitivity only to the relative phases of the various contributions, the phase of $H_{0}^{K^{*}(892)}$ is fixed to zero. The detector resolution in $M_{K \pi}$ and $M_{\psi^{\prime} \pi}$ $\left(\sigma \sim 3 \mathrm{MeV} / c^{2}\right)$ is small compared to the width of any of the resonances that are considered and is ignored. The masses and widths of all the $K^{*}$ resonances except $K_{0}^{*}(800)$ are fixed to their nominal values [15]. The mass and width of $K_{0}^{*}(800)$ are fixed to the fit results in the default model without a $Z^{-}\left(\mathrm{M}=946 \pm 50 \mathrm{MeV} / c^{2}\right.$, $\Gamma=736 \pm 126 \mathrm{MeV}$ ); the case of free mass and width is included to systematic uncertainty. We do not constrain the mass and the width of the $Z(4430)^{-}$to the previously measured values [4]. The $r$ parameters in the Blatt-Weisskopf form factors are fixed at a default value of $1.6 \mathrm{GeV}^{-1}$. 


\section{RESULTS}

\section{A. Fit to the background distribution}

The background shape is determined using $\Delta E$ sidebands. The background density function is defined as

$$
B(\Phi)=P_{2}\left(M_{K^{-} \pi^{+}}^{2}, M_{\psi^{\prime} \pi^{+}}^{2}\right)
$$

where $P_{2}$ is a two-dimensional second-order polynomial. We perform an unbinned maximum likelihood fit; the function to be minimized is given by Eq. (9) with $b=1$; thus, the resulting $B(\Phi)$ is efficiency corrected. The results of the fit, projected onto the Dalitz variables, are shown in Fig. 4. If the angular variables are also considered and the fitting function is multiplied by additional polynomials $P_{2}^{(\varphi)}(\varphi)$ and $P_{2}^{(\theta)}\left(\theta_{\psi^{\prime}}\right)$, the coefficients of the nonconstant terms are consistent with zero after minimization; thus, the background does not depend on the angular variables.
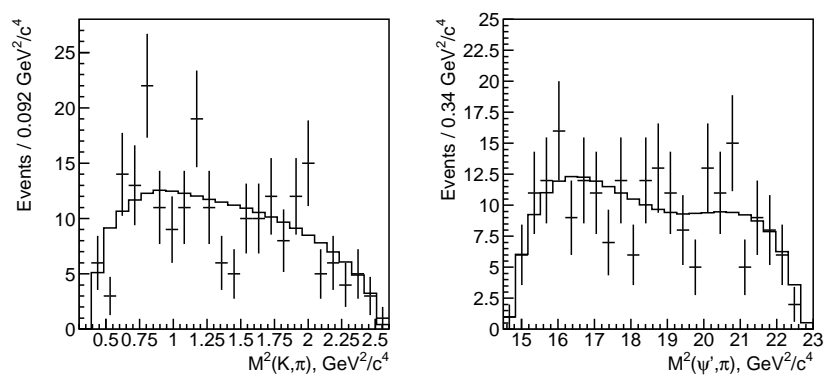

FIG. 4: The results of the fit to background events projected onto the Dalitz variables.

\section{B. Fit to the data}

The fit results for the $Z(4430)^{-}$mass, width and significance in the default model are shown in Table [ for all spin-parity hypotheses with $J \leq 2$. Note that the $0^{+}$assignment is forbidden by parity conservation in $Z(4430)^{-} \rightarrow \psi^{\prime} \pi^{-}$decays. The significance of the $Z(4430)^{-}$is estimated from the difference of $-2 \ln L$ between the models with and without a $Z(4430)^{-}$signal, taking into account the number of added degrees of freedom (6 for the $1^{+}$and $2^{-}$hypotheses or 4 for other hypotheses). The preferred $Z(4430)^{-}$spin-parity hypothesis is $1^{+}$. To test the goodness of fit we bin the Dalitz distribution with the requirement that the number of events in each bin $n_{i}>16$. We then calculate the $\chi^{2}$ value as $\sum_{i}\left(n_{i}-s_{i}\right)^{2} / s_{i}$, where $s_{i}$ is the integral of the fitting function over the bin $i$. We generate MC pseudoexperiments in accordance with the result of the fit; the confidence level is defined as the fraction of the pseudoexperiments with the $\chi^{2}$ value greater than the $\chi^{2}$ value in data. The confidence level of the $1^{+}$hypothesis is $15 \%$. The absolute values and phases of the amplitudes for the $1^{+}$ hypothesis are listed in Table II. The significances of the $K^{*}$ resonances are shown in Table III.

To present the fit results, the Dalitz distribution is divided into slices that are shown in Fig. 5. The second and fourth vertical slices correspond to the regions of the $K^{*}(892)$ and $K_{2}^{*}(1430)$, respectively; the second horizontal slice corresponds to the region of the $Z(4430)^{-}$. Projections of the fit results onto $M_{K \pi}^{2}$ and $M_{\psi^{\prime} \pi}^{2}$ axes

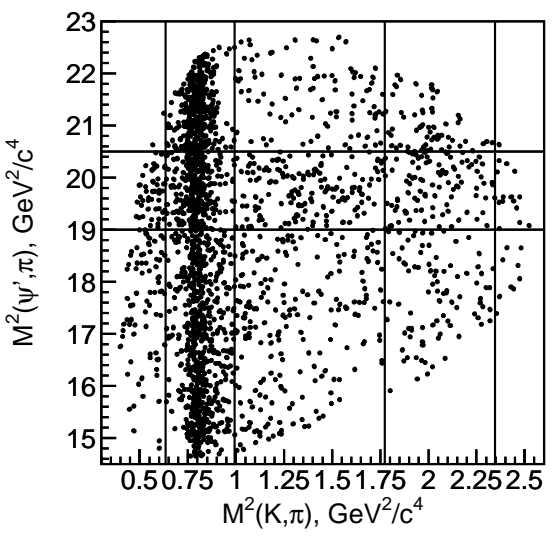

FIG. 5: Dalitz plot slices used to present fit results. Vertical divisions are at $(0.796)^{2} \mathrm{GeV}^{2} / c^{4},(0.996)^{2} \mathrm{GeV}^{2} / c^{4}$, $(1.332)^{2} \mathrm{GeV}^{2} / c^{4}$ and $(1.532)^{2} \mathrm{GeV}^{2} / c^{4}$. Horizontal divisions are at $19.0 \mathrm{GeV}^{2} / c^{4}$ and $20.5 \mathrm{GeV}^{2} / c^{4}$.

for the $1^{+}$hypothesis and the model without $Z(4430)^{-}$ are shown in Fig. 6. The sum of the first, third and fifth vertical slices [the $M^{2}\left(\psi^{\prime} \pi\right)$ projection with the $K^{*}$ veto applied] is shown in Fig. 7. Projections onto the angular variables are shown in Fig. 8.

We also consider other amplitude models, including models without one of the insignificant $K^{*}$ resonances $\left[K^{*}(1410), K^{*}{ }_{0}(1430), K^{*}(1680)\right]$; with the addition of nonresonant $K^{+} \pi^{-}$amplitudes in S, P and D waves; with free Blatt-Weisskopf $r$ parameters; with free masses and widths of $K^{*}$ resonances (within their uncertainties [15]) and with LASS amplitudes [18] instead of Breit-Wigner amplitudes for all spin-0 $K^{*}$ resonances.

In Ref. [4], the assigned value of the $B$-decay orbital angular momentum $(L)$ is varied to study the systematic uncertainty. In this analysis, we instead change the default helicity amplitudes with minimal $L$ to partial wave amplitudes with known $L$ (which does not result in a significant improvement of the likelihood); this model is also included in the systematic uncertainty.

The significances of the $Z(4430)^{-}$for all models other than the default one are shown in Table IV. The significance of the $1^{+}$hypothesis is above $5.0 \sigma$ for all the models.

The exclusion levels of the spin-parity hypotheses $\left(J^{P}=j^{p}\right)$ are calculated from MC simulation. For 
TABLE I: Fit results in the default model. Errors are statistical only.

\begin{tabular}{c|c|c|c|c|c}
\hline \hline$J^{P}$ & $0^{-}$ & $1^{-}$ & $1^{+}$ & $2^{-}$ & $2^{+}$ \\
\hline Mass, MeV $/ c^{2}$ & $4479 \pm 16$ & $4477 \pm 4$ & $4485 \pm 20$ & $4478 \pm 22$ & $4384 \pm 19$ \\
Width, MeV & $110 \pm 50$ & $22 \pm 14$ & $200 \pm 40$ & $83 \pm 25$ & $52 \pm 28$ \\
Significance & $4.5 \sigma$ & $3.6 \sigma$ & $6.4 \sigma$ & $2.2 \sigma$ & $1.8 \sigma$ \\
\hline \hline
\end{tabular}

TABLE II: The absolute values and phases of the amplitudes in the default model for the $1^{+}$spin-parity of the $Z(4430)^{-}$. Errors are statistical only.

\begin{tabular}{c|c|c|c|c|c|c}
\hline \hline Resonance & $a_{0}$ & $\phi_{0}$ & $a_{1}$ & $\phi_{1}$ & $a_{-1}$ & $\phi_{-1}$ \\
\hline$K_{0}^{*}(800)$ & $2.03 \pm 0.44$ & $1.87 \pm 0.22$ & $\ldots$ & $\ldots$ & $\ldots$ & $\ldots$ \\
$K^{*}(892)$ & 1 (fixed) & 0 (fixed) & $0.81 \pm 0.07$ & $-2.79 \pm 0.12$ & $0.43 \pm 0.08$ & $-1.64 \pm 0.15$ \\
$K^{*}(1410)$ & $0.52 \pm 0.22$ & $0.12 \pm 0.66$ & $0.47 \pm 0.44$ & $-1.38 \pm 0.55$ & $0.57 \pm 0.31$ & $1.38 \pm 0.66$ \\
$K_{0}^{*}(1430)$ & $1.08 \pm 0.50$ & $-2.57 \pm 0.63$ & $\ldots$ & $\ldots$ & $\ldots$ & $\ldots$ \\
$K_{2}^{*}(1430)$ & $8.48 \pm 2.45$ & $-0.41 \pm 0.33$ & $12.6 \pm 4.2$ & $2.56 \pm 0.69$ & $6.44 \pm 4.21$ & $-2.44 \pm 1.12$ \\
$K^{*}(1680)$ & $0.31 \pm 0.51$ & $2.08 \pm 0.17$ & $1.91 \pm 0.77$ & $3.08 \pm 0.26$ & $0.48 \pm 0.59$ & $-1.94 \pm 2.03$ \\
$Z(4430)^{-}$ & $8.85 \pm 2.57$ & $-2.97 \pm 0.77$ & $8.83 \pm 2.75$ & $-2.80 \pm 0.27$ & $\left(a_{-1} e^{i \phi_{-1}}\right)=\left(a_{1} e^{i \phi_{1}}\right)$ \\
\hline \hline
\end{tabular}

TABLE III: The fit fractions and significances of all resonances in the default model $\left(J^{P}=1^{+}\right)$.

\begin{tabular}{c|c|c}
\hline \hline Resonance & Fit fraction & Significance \\
\hline$K_{0}^{*}(800)$ & $(5.8 \pm 2.1) \%$ & $3.6 \sigma$ \\
$K^{*}(892)$ & $(63.8 \pm 2.6) \%$ & $43.1 \sigma$ \\
$K^{*}(1410)$ & $(4.3 \pm 2.3) \%$ & $0.6 \sigma$ \\
$K_{0}^{*}(1430)$ & $(1.1 \pm 1.4) \%$ & $1.6 \sigma$ \\
$K_{2}^{*}(1430)$ & $(4.5 \pm 1.0) \%$ & $3.3 \sigma$ \\
$K^{*}(1680)$ & $(4.4 \pm 1.9) \%$ & $1.0 \sigma$ \\
$Z(4430)^{-}$ & $\left(10.3_{-3.5}^{+3.0}\right) \%$ & $6.4 \sigma$ \\
\hline \hline
\end{tabular}

TABLE IV: Model dependence of the $Z(4430)^{-}$significance.

\begin{tabular}{c|c|c|c|c|c}
\hline \hline Model & $0^{-}$ & $1^{-}$ & $1^{+}$ & $2^{-}$ & $2^{+}$ \\
\hline Without $K^{*}(1410)$ & $3.8 \sigma$ & $3.4 \sigma$ & $6.9 \sigma$ & $2.1 \sigma$ & $1.0 \sigma$ \\
Without $K_{0}^{*}(1430)$ & $4.9 \sigma$ & $3.5 \sigma$ & $7.4 \sigma$ & $1.4 \sigma$ & $1.0 \sigma$ \\
Without $K^{*}(1680)$ & $4.2 \sigma$ & $3.3 \sigma$ & $7.2 \sigma$ & $2.6 \sigma$ & $1.4 \sigma$ \\
With $K_{3}^{*}(1780)$ & $2.9 \sigma$ & $3.1 \sigma$ & $5.2 \sigma$ & $2.2 \sigma$ & $1.6 \sigma$ \\
LASS & $4.3 \sigma$ & $3.5 \sigma$ & $6.2 \sigma$ & $2.9 \sigma$ & $1.6 \sigma$ \\
Partial wave amplitudes & $4.6 \sigma$ & $3.5 \sigma$ & $6.8 \sigma$ & $2.4 \sigma$ & $1.8 \sigma$ \\
Free masses and widths & $4.8 \sigma$ & $3.5 \sigma$ & $6.4 \sigma$ & $2.7 \sigma$ & $2.0 \sigma$ \\
Free $r$ & $4.1 \sigma$ & $3.7 \sigma$ & $6.4 \sigma$ & $2.4 \sigma$ & $1.9 \sigma$ \\
Nonresonant ampl. (S) & $5.1 \sigma$ & $3.6 \sigma$ & $6.8 \sigma$ & $2.7 \sigma$ & $1.7 \sigma$ \\
Nonresonant ampl. (S,P) & $5.4 \sigma$ & $3.6 \sigma$ & $6.9 \sigma$ & $3.0 \sigma$ & $2.2 \sigma$ \\
Nonresonant ampl. (S,P,D) & $3.6 \sigma$ & $2.7 \sigma$ & $5.6 \sigma$ & $2.2 \sigma$ & $1.4 \sigma$ \\
\hline \hline
\end{tabular}

each amplitude model, we generate MC pseudoexperiments in accordance with the fit result with the $j^{p}$ $Z(4430)^{-}$signal in data and fit them with the $j^{p}$ and $1^{+}$signals. The resulting distribution of $\Delta(-2 \ln L)=$ $(-2 \ln L)_{J^{P}=j^{p}}-(-2 \ln L)_{J^{P}=1^{+}}$is fitted to an asymmetrical Gaussian function and the $p$-value is calculated as the integral of the fitting function normalized to 1 from the value of $\Delta(-2 \ln L)$ in data to $+\infty$. The results are presented in Table VI. The $J^{P}=1^{+}$hypothesis is favored over the $0^{-}, 1^{-}, 2^{-}$and $2^{+}$hypotheses at the levels of $3.4 \sigma, 3.7 \sigma, 4.7 \sigma$ and $5.1 \sigma$, respectively.

We also generate MC pseudoexperiments in accordance with the fit results for the $1^{+}$hypothesis and obtain the distribution of $\Delta(-2 \ln L)$. This distribution is fitted to an asymmetrical Gaussian function and the confidence level of the $1^{+}$hypothesis is calculated as the integral of the fitting function normalized to 1 from $-\infty$ to the value of $\Delta(-2 \ln L)$ in data. The resulting confidence levels are shown in Table VI The distributions of $\Delta(-2 \ln L)$ for $j^{p}=0^{-}$are shown in Fig. 9 .

The results of the study of the model dependence of the $Z(4430)^{-}$mass and width are shown in Table $\mathbf{V}$. The maximal deviations of the mass and the width of the $Z(4430)^{-}$from their optimal values are considered as overall systematic uncertainty due to the amplitude model dependence. We also estimate the uncertainty due to the uncertainties of the fit to the background distribution by varying the background parameters by $\pm 1 \sigma$ (with other parameters varied in accordance with the correlation coefficients) and performing the fit to the data. The maximal deviations are considered as systematic uncertainty.

\section{Efficiency and branching fractions}

We use the signal density function determined from the fits to calculate the efficiency

$$
\epsilon_{0}=\frac{\int S(\Phi) \epsilon(\Phi) d \Phi}{\int S(\Phi) d \Phi}
$$



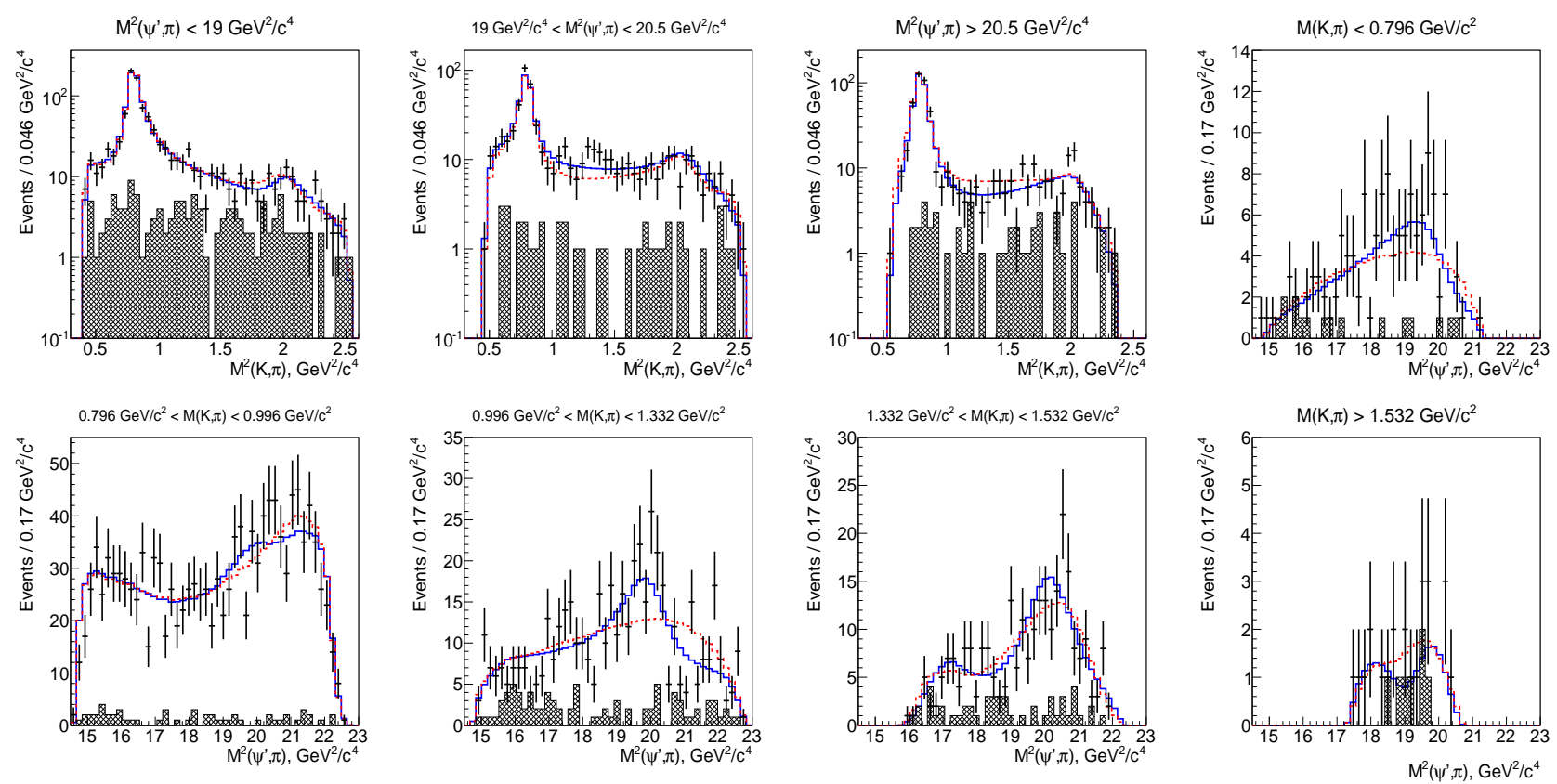

FIG. 6: The fit results with (solid line) and without (dashed line) $Z^{-}\left(J^{P}=1^{+}\right)$in the default model. The points with error bars are data; the hatched histograms are $\psi^{\prime}$ sidebands. Slices are defined in Fig. 5

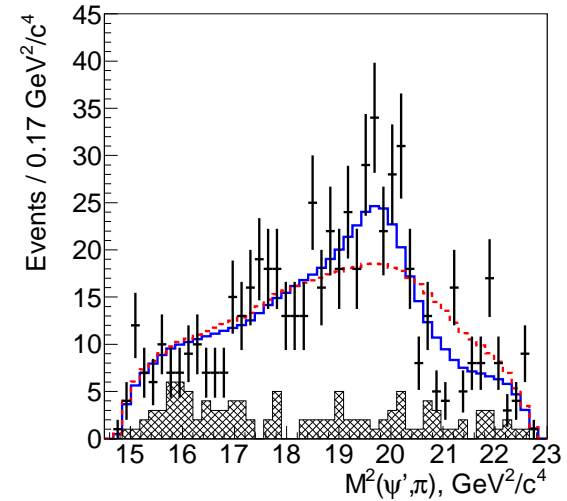

FIG. 7: Projection of the fit results with the $K^{*}$ veto. The legend is the same as in Fig. [6]

where $\epsilon(\Phi)$ is the phase-space-dependent efficiency. The reconstruction efficiency is found to be $(28.3 \pm 1.2) \%$. The central value is calculated for the default model with $Z^{-}$ $\left(J^{P}=1^{+}\right)$. The error includes the uncertainty in track reconstruction efficiency $(1.4 \%)$, the error from the particle identification efficiency difference between MC and data $(3.8 \%)$ and the uncertainty due to the amplitude model dependence $(0.5 \%)$. The error due to MC statistics is negligibly small. The efficiency includes the correction for the difference between the particle identification efficiency in MC and data, $(94.2 \pm 3.5) \%$.

Using the obtained efficiency and the branching frac-
TABLE V: Systematic uncertainties in the $Z(4430)^{-}$mass (in $\mathrm{MeV} / c^{2}$ ) and width (in $\mathrm{MeV}$ ).

\begin{tabular}{c|c|c}
\hline \hline Model or error source & Mass & Width \\
\hline Without $K^{*}(1410)$ & ${ }_{-0}^{+4}$ & ${ }_{-9}^{+0}$ \\
Without $K_{0}^{*}(1430)$ & ${ }_{-0}^{+18}$ & ${ }_{-0}^{+24}$ \\
Without $K^{*}(1680)$ & ${ }_{-0}^{+27}$ & ${ }_{-32}^{+0}$ \\
With $K_{3}^{*}(1780)$ & ${ }_{-0}^{+5}$ & ${ }_{-0}^{+23}$ \\
LASS & ${ }_{-0}^{+0}$ & ${ }_{-3}^{+13}$ \\
Partial wave amplitudes & ${ }_{-0}^{+12}$ & ${ }_{-0}^{+0}$ \\
Free masses and widths & ${ }_{-1}^{+0}$ & ${ }_{-2}^{+0}$ \\
Free $r$ & ${ }_{-0}^{+13}$ & ${ }_{-0}^{+9}$ \\
Nonresonant ampl. (S) & ${ }_{-0}^{+0}$ & ${ }_{-0}^{+13}$ \\
Nonresonant ampl. (S,P) & ${ }_{-1}^{+0}$ & ${ }_{-0}^{+8}$ \\
Nonresonant ampl. (S,P,D) & ${ }_{-0}^{+2}$ & ${ }_{-0}^{+9}$ \\
\hline Amplitude model, total & ${ }_{-11}^{+27}$ & ${ }_{-32}^{+24}$ \\
Background & ${ }_{-1}^{+2}$ & ${ }_{-9}^{+3}$ \\
\hline Total & ${ }_{-11}^{+27}$ & ${ }_{-33}^{+24}$ \\
\hline \hline
\end{tabular}

tions of $\psi^{\prime}$ decays to $e^{+} e^{-}$and $\mu^{+} \mu^{-}[15]$, we determine:

$$
\begin{gathered}
\mathcal{B}\left(B^{0} \rightarrow \psi^{\prime} K^{+} \pi^{-}\right) \times \mathcal{B}\left(\psi^{\prime} \rightarrow \ell^{+} \ell^{-}\right)= \\
(9.12 \pm 0.30 \pm 0.51) \times 10^{-6}
\end{gathered}
$$

and

$$
\mathcal{B}\left(B^{0} \rightarrow \psi^{\prime} K^{+} \pi^{-}\right)=(5.90 \pm 0.20 \pm 0.36) \times 10^{-4} .
$$

This result assumes equal production of $B^{0} \bar{B}^{0}$ and $B^{+} B^{-}$pairs. The systematic error includes the uncer- 
TABLE VI: Exclusion levels of spin-parity hypotheses and confidence levels of the $1^{+}$hypothesis.

\begin{tabular}{c|c|c|c|c|c|c|c|c}
\hline \hline \multirow{2}{*}{ Model } & \multicolumn{2}{|c|}{$0^{-}$} & \multicolumn{2}{|c|}{$1^{-}$} & \multicolumn{2}{c|}{$2^{-}$} & \multicolumn{2}{c}{$2^{+}$} \\
\cline { 2 - 8 } & $1^{+}$over $0^{-}$ & $1^{+}$C.L. & $1^{+}$over $1^{-}$ & $1^{+}$C.L. & $1^{+}$over $2^{-}$ & $1^{+}$C.L. & $1^{+}$over $2^{+}$ & $1^{+}$C.L. \\
\hline Default & $4.7 \sigma$ & $17 \%$ & $6.3 \sigma$ & $16 \%$ & $6.5 \sigma$ & $50 \%$ & $8.2 \sigma$ & $38 \%$ \\
Without $K^{*}(1410)$ & $6.4 \sigma$ & $40 \%$ & $7.2 \sigma$ & $25 \%$ & $7.7 \sigma$ & $43 \%$ & $9.2 \sigma$ & $50 \%$ \\
Without $K_{0}^{*}(1430)$ & $5.0 \sigma$ & $22 \%$ & $4.1 \sigma$ & $19 \%$ & $8.9 \sigma$ & $69 \%$ & $8.9 \sigma$ & $33 \%$ \\
Without $K^{*}(1680)$ & $7.1 \sigma$ & $54 \%$ & $8.2 \sigma$ & $58 \%$ & $10.0 \sigma$ & $79 \%$ & $11.1 \sigma$ & $75 \%$ \\
With $K_{3}^{*}(1780)$ & $3.4 \sigma$ & $53 \%$ & $3.7 \sigma$ & $9.8 \%$ & $4.7 \sigma$ & $27 \%$ & $5.1 \sigma$ & $29 \%$ \\
LASS & $4.8 \sigma$ & $9.7 \%$ & $6.3 \sigma$ & $12 \%$ & $5.5 \sigma$ & $28 \%$ & $8.2 \sigma$ & $30 \%$ \\
Partial wave amplitudes & $5.1 \sigma$ & $30 \%$ & $6.6 \sigma$ & $28 \%$ & $7.6 \sigma$ & $52 \%$ & $9.7 \sigma$ & $46 \%$ \\
Free masses and widths & $4.8 \sigma$ & $15 \%$ & $6.0 \sigma$ & $14 \%$ & $6.3 \sigma$ & $37 \%$ & $7.4 \sigma$ & $35 \%$ \\
Free $r$ & $5.5 \sigma$ & $19 \%$ & $5.7 \sigma$ & $26 \%$ & $6.5 \sigma$ & $37 \%$ & $7.3 \sigma$ & $43 \%$ \\
Nonresonant ampl. (S) & $3.9 \sigma$ & $18 \%$ & $5.0 \sigma$ & $9.3 \%$ & $6.1 \sigma$ & $38 \%$ & $8.4 \sigma$ & $25 \%$ \\
Nonresonant ampl. (S,P) & $3.4 \sigma$ & $20 \%$ & $5.0 \sigma$ & $18 \%$ & $6.2 \sigma$ & $46 \%$ & $6.2 \sigma$ & $34 \%$ \\
Nonresonant ampl. (S,P,D) & $3.8 \sigma$ & $20 \%$ & $4.8 \sigma$ & $14 \%$ & $5.2 \sigma$ & $41 \%$ & $5.2 \sigma$ & $26 \%$ \\
\hline \hline
\end{tabular}

tainty in the efficiency, the number of $B$ mesons (1.4\%), the signal yield $(3.7 \%)$ and the $\psi^{\prime} \rightarrow \ell^{+} \ell^{-}$branching fraction (2.2\% assuming lepton universality). This result is combined with the value of the same branching fraction measured in the $\psi^{\prime} \rightarrow J / \psi \pi^{+} \pi^{-}$channel in Ref. [4], taking into account the correlations between the error sources. The final combined result is

$$
\mathcal{B}\left(B^{0} \rightarrow \psi^{\prime} K^{+} \pi^{-}\right)=(5.80 \pm 0.39) \times 10^{-4},
$$

where the uncertainty includes statistical and systematic errors. As we perform a full amplitude analysis, the contributions of the individual resonances are described more precisely than in Ref. [4], and we do not combine the results of the measurements below.

The fit fraction of a resonance $R$ [the $Z(4430)^{-}$or one of the $K^{*}$ resonances] is defined as

$$
f=\frac{\int S_{R}(\Phi) d \Phi}{\int S(\Phi) d \Phi}
$$

where $S_{R}(\Phi)$ is the signal density function with all contributions other than the contribution of the $R$ resonance set to 0 . The statistical uncertainties in the fit fractions are determined from a set of MC pseudoexperiments generated in accordance with the fit result in data. We fit each sample and calculate the fit fractions; the resulting distribution of the fit fractions is fitted to a Gaussian function, and the sigma of the Gaussian function is treated as the statistical uncertainty. The results are summarized in Table III.

Using the fit fraction of the $K^{*}(892)$ and the combined $\mathcal{B}\left(B^{0} \rightarrow \psi^{\prime} K^{+} \pi^{-}\right)$, we calculate the branching fraction of $B^{0} \rightarrow \psi^{\prime} K^{*}(892)$ decay:

$$
\mathcal{B}\left(B^{0} \rightarrow \psi^{\prime} K^{*}(892)\right)=\left(5.55_{-0.23-0.84}^{+0.22+0.41}\right) \times 10^{-4} .
$$

The central value is given for the default model with the $Z(4430)^{-}$having $J^{P}=1^{+}$. The systematic error includes contributions from the same sources as the uncertainty in the branching fraction of the $B^{0} \rightarrow$ $\psi^{\prime} K^{+} \pi^{-}$decay, the amplitude model $\left[\left({ }_{-13.0}^{+4.8}\right) \%\right]$ and the background parametrization $\left[\left({ }_{-5.5}^{+0.8}\right) \%\right]$ dependence of the $K^{*}(892)$ fit fraction. We also determine the fraction of the $K^{*}(892)$ mesons that are longitudinally polarized: $f_{L}=\left(45.5_{-2.9-4.9}^{+3.1+1.4}\right) \%$.

The branching fraction product for the $Z(4430)^{-}$is

$$
\begin{aligned}
& \mathcal{B}\left(B^{0} \rightarrow Z(4430)^{-} K^{+}\right) \times \mathcal{B}\left(Z(4430)^{-} \rightarrow \psi^{\prime} \pi^{-}\right)= \\
& \quad\left(6.0_{-2.0-1.4}^{+1.7+2.5}\right) \times 10^{-5},
\end{aligned}
$$

where the systematic error due to the amplitude model dependence is $\left({ }_{-22.4}^{+41.2}\right) \%$ and the systematic error due to the background parametrization dependence is $\left({ }_{-3.5}^{+3.1}\right) \%$.

\section{CONCLUSIONS}

We have performed an amplitude analysis of $B^{0} \rightarrow$ $\psi^{\prime} K^{+} \pi^{-}$decays in four dimensions. The preferred assignment of the quantum numbers of the $Z(4430)^{-}$is $1^{+}$. The $J^{P}=1^{+}$hypothesis is favored over the $0^{-}, 1^{-}$, $2^{-}$and $2^{+}$hypotheses at the levels of $3.4 \sigma, 3.7 \sigma, 4.7 \sigma$ and $5.1 \sigma$, respectively. The results for the mass and the width of the $Z(4430)^{-}$are

$$
\begin{aligned}
M & =4485_{-22-11}^{+22+28} \mathrm{MeV} / c^{2}, \\
\Gamma & =200_{-46-35}^{+41+26} \mathrm{MeV} .
\end{aligned}
$$

We calculate the branching fractions to be

$$
\begin{gathered}
\mathcal{B}\left(B^{0} \rightarrow \psi^{\prime} K^{+} \pi^{-}\right)=(5.80 \pm 0.39) \times 10^{-4}, \\
\mathcal{B}\left(B^{0} \rightarrow \psi^{\prime} K^{*}(892)\right)=\left(5.55_{-0.23-0.84}^{+0.22+0.41}\right) \times 10^{-4}, \\
\mathcal{B}\left(B^{0} \rightarrow Z(4430)^{-} K^{+}\right) \times \mathcal{B}\left(Z(4430)^{-} \rightarrow \psi^{\prime} \pi^{-}\right)= \\
\left(6.0_{-2.0-1.4}^{+1.7+2.5}\right) \times 10^{-5},
\end{gathered}
$$

and the fraction of the longitudinally polarized $K^{*}(892)$ mesons to be

$$
f_{L}=\left(45.5_{-2.9-4.9}^{+3.1+1.4}\right) \% \text {. }
$$



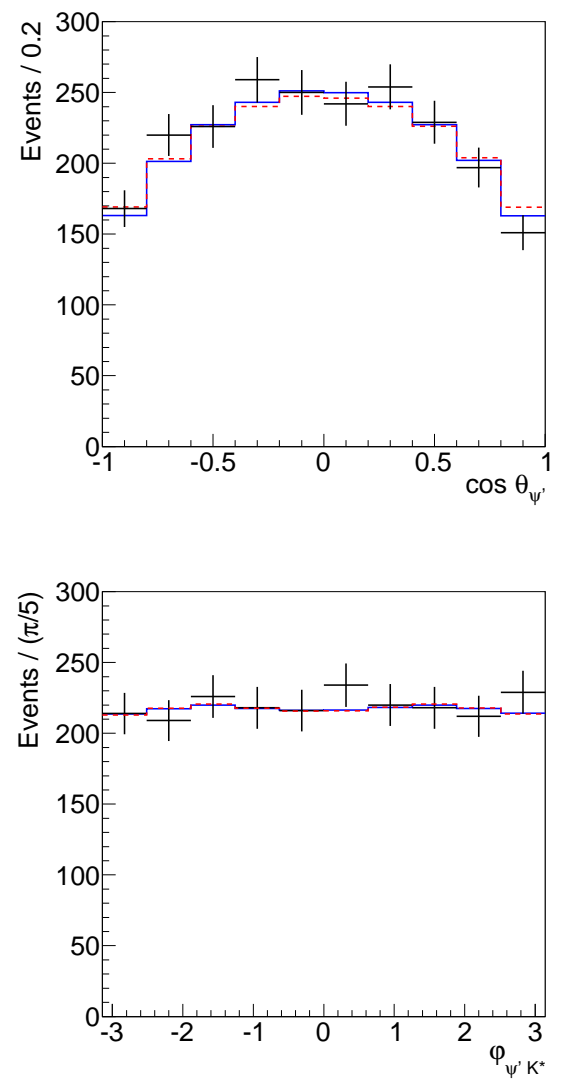

FIG. 8: Projections of the fit results with (solid line) and without (dashed line) $Z^{-}\left(J^{P}=1^{+}\right)$onto the angular variables for the entire signal region (no $K^{*}$ veto) in the default model. Points with error bars are data.

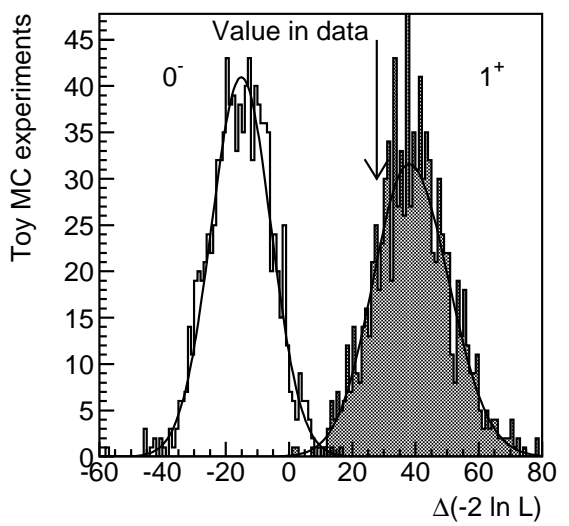

FIG. 9: Comparison of the $0^{-}$and $1^{+}$hypotheses in the default model. The histograms are distributions of $\Delta(-2 \ln L)$ in $\mathrm{MC}$ pseudoexperiments generated in accordance with the fit results with $0^{-}$(open histogram) and $1^{+}$(hatched histogram $)$ signals. The $\Delta(-2 \ln L)$ value observed in data is indicated with an arrow.
These results supersede previous measurements from a Dalitz analysis of the same decay channel [4].

\section{ACKNOWLEDGMENTS}

We thank the KEKB group for the excellent operation of the accelerator; the KEK cryogenics group for the efficient operation of the solenoid; and the KEK computer group, the National Institute of Informatics, and the PNNL/EMSL computing group for valuable computing and SINET4 network support. We acknowledge support from the Ministry of Education, Culture, Sports, Science, and Technology (MEXT) of Japan, the Japan Society for the Promotion of Science (JSPS), and the Tau-Lepton Physics Research Center of Nagoya University; the Australian Research Council and the Australian Department of Industry, Innovation, Science and Research; Austrian Science Fund under Grant No. P 22742-N16; the National Natural Science Foundation of China under contract No. 10575109, 10775142, 10875115 and 10825524; the Ministry of Education, Youth and Sports of the Czech Republic under contract No. MSM0021620859; the Carl Zeiss Foundation, the Deutsche Forschungsgemeinschaft and the VolkswagenStiftung; the Department of Science and Technology of India; the Istituto Nazionale di Fisica Nucleare of Italy; The BK21 and WCU program of the Ministry Education Science and Technology, National Research Foundation of Korea Grant No. 2010-0021174, 2011-0029457, 2012-0008143, 2012R1A1A2008330, BRL program under NRF Grant No. KRF-2011-0020333, and GSDC of the Korea Institute of Science and Technology Information; the Polish Ministry of Science and Higher Education and the National Science Center; the Ministry of Education and Science of the Russian Federation, the Russian Federal Agency for Atomic Energy and Russian Foundation for Basic Research grant 12-02-00862-a; the Slovenian Research Agency; the Basque Foundation for Science (IKERBASQUE) and the UPV/EHU under program UFI 11/55; the Swiss National Science Foundation; the National Science Council and the Ministry of Education of Taiwan; and the U.S. Department of Energy and the National Science Foundation. This work is supported by a Grant-in-Aid from MEXT for Science Research in a Priority Area ("New Development of Flavor Physics"), and from JSPS for Creative Scientific Research ("Evolution of Tau-lepton Physics"). 
[1] S. Godfrey, S. L. Olsen, Ann. Rev. Nucl. Part. Sci. 58, 51 (2008).

[2] N. Brambilla et al., Eur. Phys. J. C 71, 1534 (2011).

[3] S. K. Choi et al. (Belle Collaboration), Phys. Rev. Lett. 100, 142001 (2008).

[4] R. Mizuk et al. (Belle Collaboration), Phys. Rev. D 80, 031104(R) (2009).

[5] R. Mizuk et al. (Belle Collaboration), Phys. Rev. D 78, 072004 (2008).

[6] B. Aubert et al. (BaBar Collaboration), Phys. Rev. D 79, 112001 (2009).

[7] J. P. Lees et al. (BaBar Collaboration), Phys. Rev. D 85 052003 (2012).

[8] M. Ablikim et al. (BESIII Collaboration), Phys. Rev. Lett. 110, 252001 (2013).

[9] Z. Q. Liu et al. (Belle Collaboration), Phys. Rev. Lett. 110, 252002 (2013).

[10] S. Kurokawa and E. Kikutani, Nucl. Instrum. Methods Phys. Res. Sect. A 499, 1 (2003), and other papers included in this Volume; T. Abe et al., Prog. Theor. Exp. Phys. 2013, 03 A001 (2013) and following articles up to $03 \mathrm{~A} 011$.

[11] A. Abashian et al. (Belle Collaboration), Nucl. Instrum. Methods Phys. Res. Sect. A 479, 117 (2002); also see detector section in J. Brodzicka et al., Prog. Theor. Exp. Phys. 2012, 04D001 (2012).

[12] Z. Natkaniec et al. (Belle SVD2 Group), Nucl. Instrum. Methods Phys. Res. Sect. A 560, 1 (2006).

[13] R. Brun et al., GEANT 3.21, CERN DD/EE/84-1, 1984.

[14] D. J. Lange, Nucl. Instrum. Methods Phys. Res. Sect. A462, 152 (2001).

[15] J. Beringer et al. (Particle Data Group), Phys. Rev. D 86, 010001 (2012).

[16] J. Blatt and V. Weisskopf, Theoretical Nuclear Physics (John Wiley \& Sons, New York, 1952), p. 361.

[17] A. Garmash et al. (Belle Collaboration), Phys. Rev. D 71, 092003 (2005).

[18] D. Aston et al., Nucl. Phys. B 296, 493 (1988).

\section{APPENDIX: DERIVATION OF THE SIGNAL DENSITY FUNCTION}

\section{A. Decay via the $K^{*}$ resonances}

The definition of the angle between the decay planes of the $\psi^{\prime}$ and $K^{*}$ is shown in Fig. 10, The coordinate systems $\left(x_{K^{*}}, y_{K^{*}}, z_{K^{*}}\right)$ and $\left(x_{\psi^{\prime}}, y_{\psi^{\prime}}, z_{\psi^{\prime}}\right)$ are defined in the $B^{0}$ rest frame; the $x_{K^{*}}$ and $x_{\psi^{\prime}}$ axes are chosen to be the same. The angle $\phi$ is given by

$$
\phi=\phi_{K^{+}}+\phi_{\ell^{+}}
$$

where $\phi_{K^{+}}$and $\phi_{\ell^{+}}$are the azimuthal angles of the $K^{+}$ and $\ell^{+}$, respectively. This angle may be calculated as

$$
\begin{aligned}
& \cos \varphi=\frac{\left(\vec{a}_{K^{+}} \cdot \vec{a}_{\ell^{+}}\right)}{\left|\vec{a}_{K^{+}}\right|\left|\vec{a}_{\ell^{+}}\right|}, \\
& \sin \varphi=\frac{\left(\left[\vec{p}_{\psi^{\prime}} \times \vec{a}_{K^{+}}\right] \cdot \vec{a}_{\ell^{+}}\right)}{\left|\vec{p}_{\psi^{\prime}}\right|\left|\vec{a}_{K^{+}}\right|\left|\vec{a}_{\ell^{+}}\right|},
\end{aligned}
$$

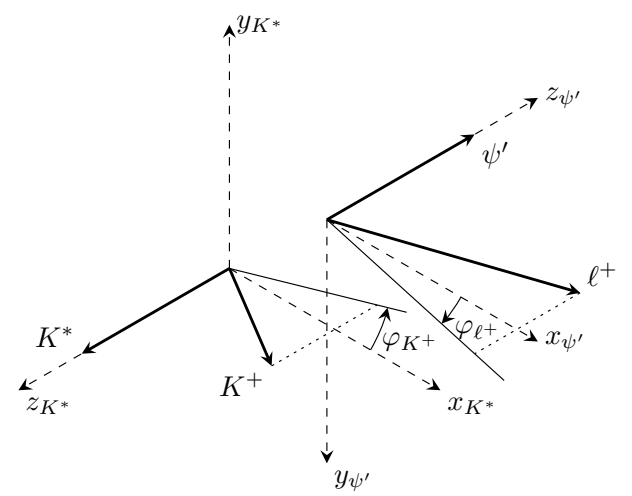

FIG. 10: Definition of the angle between the decay planes for $B^{0} \rightarrow \psi^{\prime}\left(\rightarrow \ell^{+} \ell^{-}\right) K^{*}\left(\rightarrow K^{+} \pi^{-}\right)$decay (in the $B^{0}$ rest frame).

where

$$
\begin{aligned}
\vec{a}_{K^{+}} & =\vec{p}_{K^{+}}-\frac{\left(\vec{p}_{K^{+}} \cdot \vec{p}_{K^{*}}\right)}{\left|\vec{p}_{K^{*}}\right|^{2}} \vec{p}_{K^{*}}, \\
\vec{a}_{\ell^{+}} & =\vec{p}_{\ell^{+}}-\frac{\left(\vec{p}_{\ell^{+}} \cdot \vec{p}_{\psi^{\prime}}\right)}{\left|\vec{p}_{\psi^{\prime}}\right|^{2}} \vec{p}_{\psi^{\prime}},
\end{aligned}
$$

where $\vec{p}_{K^{+}}, \vec{p}_{K^{*}}, \vec{p}_{\ell^{+}}$and $\vec{p}_{\psi^{\prime}}$ are the momenta of $K^{+}$, $K^{*}, \ell^{+}$and $\psi^{\prime}$ in the $B^{0}$ rest frame, respectively.

The definitions of the helicity angles are shown in Fig. 11] The coordinate systems $\left(x_{K^{*}}^{\prime}, y_{K^{*}}^{\prime}, z_{K^{*}}^{\prime}\right)$ and $\left(x_{\psi^{\prime}}^{\prime}, y_{\psi^{\prime}}^{\prime}, z_{\psi^{\prime}}^{\prime}\right)$ are obtained by the boosting of the coordinate systems $\left(x_{K^{*}}, y_{K^{*}}, z_{K^{*}}\right)$ and $\left(x_{\psi^{\prime}}, y_{\psi^{\prime}}, z_{\psi^{\prime}}\right)$ to the rest frames of the $K^{*}$ and $\psi^{\prime}$, respectively. The $K^{*}$ he-

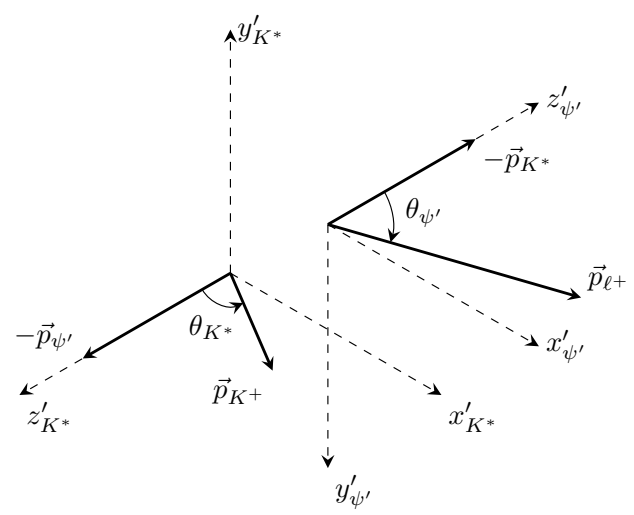

FIG. 11: Definition of the helicity angles for $B^{0} \rightarrow \psi^{\prime}(\rightarrow$ $\left.\ell^{+} \ell^{-}\right) K^{*}\left(\rightarrow K^{+} \pi^{-}\right)$decay (in the $K^{*}$ and $\psi^{\prime}$ rest frames).

licity angle is given by

$$
\cos \theta_{K^{*}}=\frac{-\left(\vec{p}_{\psi^{\prime}} \cdot \vec{p}_{K^{+}}\right)}{\left|\vec{p}_{\psi^{\prime}}\right|\left|\vec{p}_{K^{+}}\right|}
$$


where $\vec{p}_{\psi^{\prime}}$ and $\vec{p}_{K^{+}}$are the momenta of $\psi^{\prime}$ and $K^{+}$in the $K^{*}$ rest frame, respectively; the $\psi^{\prime}$ helicity angle is calculated similarly.

The amplitude of the decay $B^{0} \rightarrow \psi^{\prime}\left(\rightarrow \ell^{+} \ell^{-}\right) K^{*}(\rightarrow$ $\left.K^{+} \pi^{-}\right)$is

$$
\begin{aligned}
A_{\lambda \xi}^{K^{*}}(\Phi) & =H_{\lambda}^{K^{*}} D_{\lambda 0}^{J\left(K^{*}\right) *}\left(\varphi_{K^{+}}, \theta_{K^{*}}, 0\right) D_{\lambda \xi}^{1 *}\left(\varphi_{\ell^{+}}, \theta_{\psi^{\prime}}, 0\right) \\
& =H_{\lambda}^{K^{*}} e^{i \lambda \varphi_{K^{+}}} d_{\lambda 0}^{J\left(K^{*}\right)}\left(\theta_{K^{*}}\right) e^{i \lambda \varphi_{\ell^{+}}} d_{\lambda \xi}^{1}\left(\theta_{\psi^{\prime}}\right) \\
& =H_{\lambda}^{K^{*}} e^{i \lambda \varphi} d_{\lambda 0}^{J\left(K^{*}\right)}\left(\theta_{K^{*}}\right) d_{\lambda \xi}^{1}\left(\theta_{\psi^{\prime}}\right)
\end{aligned}
$$

where $H_{\lambda}^{K^{*}}$ is the helicity amplitude, $\lambda$ is the helicity of the $\psi^{\prime}$ and $\xi$ is the helicity of the lepton pair. Note that the orientation of the coordinate system $\left(x_{\psi^{\prime}}^{\prime \prime}, y_{\psi^{\prime}}^{\prime \prime}, z_{\psi^{\prime}}^{\prime \prime}\right)$ [this is the coordinate system $\left(x_{\psi^{\prime}}^{\prime}, y_{\psi^{\prime}}^{\prime}, z_{\psi^{\prime}}^{\prime}\right)$ rotated by $\varphi_{\ell^{+}}$around the $z$ axis and then by $\theta_{\psi^{\prime}}$ around the $y$ axis] is fixed by the condition that the $K^{*}$ momentum is lying in the plane $\left(x_{\psi^{\prime}}^{\prime \prime}, z_{\psi^{\prime}}^{\prime \prime}\right)$.

\section{B. Decay via the $Z(4430)^{-}$}

The definition of the $Z^{-}$helicity angle is shown in Fig. 12. The coordinate system $\left(x_{Z^{-}}, y_{Z^{-}}, z_{Z^{-}}\right)$is defined in the $Z(4430)^{-}$rest frame and its orientation is chosen so that the $\psi^{\prime}$ momentum is lying in the plane $\left(x_{Z^{-}}, y_{Z^{-}}\right)$.

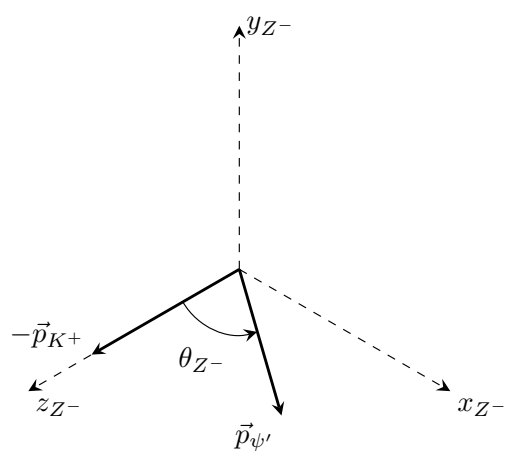

FIG. 12: Definition of the $Z(4430)^{-}$helicity angle (in the $Z(4430)^{-}$rest frame).

The definitions of the $\psi^{\prime}$ helicity angle and the angle $\tilde{\varphi}$ are shown in Fig. 13. The coordinate system $\left(\tilde{x}_{\psi^{\prime}}, \tilde{y}_{\psi^{\prime}}, \tilde{z}_{\psi^{\prime}}\right)$ is defined in the $\psi^{\prime}$ rest frame; the $K^{+}$ momentum is lying in the plane $\left(\tilde{x}_{\psi^{\prime}}, \tilde{z}_{\psi^{\prime}}\right)$, the azimuthal angle being equal to 0 . The azimuthal angle $\tilde{\varphi}$ may be calculated as

$$
\begin{aligned}
& \cos \tilde{\varphi}=\frac{\left(\vec{a}_{K^{+}} \cdot \vec{a}_{\ell^{+}}\right)}{\left|\vec{a}_{K^{+}}\right|\left|\vec{a}_{\ell^{+}}\right|}, \\
& \sin \tilde{\varphi}=\frac{-\left(\left[\vec{p}_{\pi^{-}} \times \vec{a}_{K^{+}}\right] \cdot \vec{a}_{\ell^{+}}\right)}{\left|\vec{p}_{\pi^{-}}\right|\left|\vec{a}_{K^{+}}\right|\left|\vec{a}_{\ell^{+}}\right|},
\end{aligned}
$$

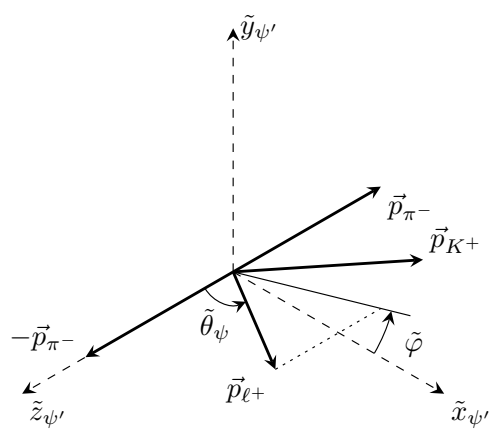

FIG. 13: Definitions of the $\psi^{\prime}$ helicity angle and the angle $\tilde{\varphi}$ (the $\psi^{\prime}$ rest frame).

where

$$
\begin{gathered}
\vec{a}_{K^{+}}=\vec{p}_{K^{+}}-\frac{\left(\vec{p}_{K^{+}} \cdot \vec{p}_{\pi^{-}}\right)}{\left|\vec{p}_{\pi^{-}}\right|^{2}} \vec{p}_{\pi^{-}}, \\
\vec{a}_{\ell^{+}}=\vec{p}_{\ell^{+}}-\frac{\left(\vec{p}_{\ell^{+}} \cdot \vec{p}_{\pi^{-}}\right)}{\left|\vec{p}_{\pi^{-}}\right|^{2}} \vec{p}_{\pi^{-}},
\end{gathered}
$$

where $\vec{p}_{K^{+}}, \vec{p}_{\pi^{-}}$and $\vec{p}_{\ell^{+}}$are the momenta of $K^{+}, \pi^{-}$and $\ell^{+}$in the $\psi^{\prime}$ rest frame, respectively.

The orientation of the coordinate system $\left(\tilde{x}_{\psi^{\prime}}^{\prime}, \tilde{y}_{\psi^{\prime}}^{\prime}, \tilde{z}_{\psi^{\prime}}^{\prime}\right)$ [this is the coordinate system $\left(\tilde{x}_{\psi^{\prime}}, \tilde{y}_{\psi^{\prime}}, \tilde{z}_{\psi^{\prime}}\right)$ rotated by $\tilde{\varphi}$ around the $z$ axis and then by $\tilde{\theta}_{\psi^{\prime}}$ around the $y$ axis] satisfies the condition that the $\pi^{-}$momentum is lying in the plane $\left(\tilde{x}_{\psi^{\prime}}^{\prime}, \tilde{z}_{\psi^{\prime}}^{\prime}\right)$; thus, this coordinate system is not the same as $\left(x_{\psi^{\prime}}^{\prime \prime}, y_{\psi^{\prime}}^{\prime \prime}, z_{\psi^{\prime}}^{\prime \prime}\right)$. The coordinate systems in question are shown in Fig. 14. The azimuthal angle $\alpha$

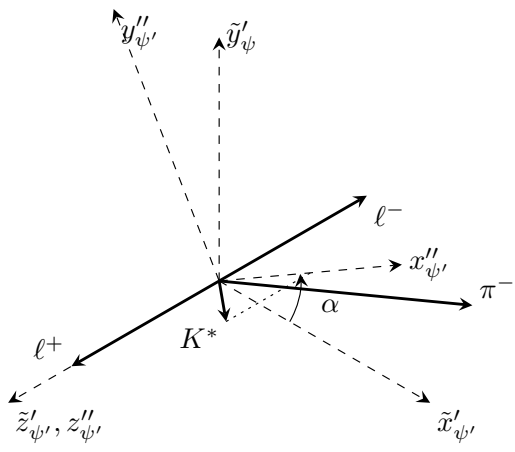

FIG. 14: Definition of the angle $\alpha$ (in the $\psi^{\prime}$ rest frame). 
may be calculated as

$$
\begin{aligned}
& \cos \alpha=\frac{\left(\vec{a}_{\pi^{-}} \cdot \vec{a}_{K^{*}}\right)}{\left|\vec{a}_{\pi^{-}}\right|\left|\vec{a}_{K^{*}}\right|}, \\
& \sin \alpha=\frac{\left(\left[\vec{p}_{\ell^{+}} \times \vec{a}_{\pi^{-}}\right] \cdot \vec{a}_{K^{*}}\right)}{\left|\vec{p}_{\ell^{+}}\right|\left|\vec{a}_{\pi^{-}}\right|\left|\vec{a}_{K^{*}}\right|},
\end{aligned}
$$

where

$$
\begin{aligned}
& \vec{a}_{K^{*}}=\vec{p}_{K^{*}}-\frac{\left(\vec{p}_{K^{*}} \cdot \vec{p}_{\ell^{+}}\right)}{\left|\vec{p}_{\ell^{+}}\right|^{2}} \vec{p}_{\ell^{+}}, \\
& \vec{a}_{\pi^{-}}=\vec{p}_{\pi^{-}}-\frac{\left(\vec{p}_{\pi^{-}} \cdot \vec{p}_{\ell^{+}}\right)}{\left|\vec{p}_{\ell^{+}}\right|^{2}} \vec{p}_{\ell^{+}},
\end{aligned}
$$

where $\vec{p}_{K^{*}}, \vec{p}_{\pi^{-}}$and $\vec{p}_{\ell^{+}}$are the momenta of $K^{*}, \pi^{-}$and $\ell^{+}$in the $\psi^{\prime}$ rest frame, respectively. After additional rotation by $\alpha$ around the $z$ axis, the coordinate system $\left(\tilde{x}_{\psi^{\prime}}^{\prime}, \tilde{y}_{\psi^{\prime}}^{\prime}, \tilde{z}_{\psi^{\prime}}^{\prime}\right)$ becomes the same as $\left(x_{\psi^{\prime}}^{\prime \prime}, y_{\psi^{\prime}}^{\prime \prime}, z_{\psi^{\prime}}^{\prime \prime}\right)$; thus, the final states are the same for the decays via the $K^{*}$ and $Z(4430)^{-}$.

The amplitude of the decay $B^{0} \rightarrow K^{+} Z^{-}\left(\rightarrow \psi^{\prime}(\rightarrow\right.$ $\left.\left.\ell^{+} \ell^{-}\right) \pi^{-}\right)$is

$$
\begin{aligned}
A_{\lambda^{\prime} \xi}^{Z^{-}}(\Phi) & =H_{\lambda^{\prime}}^{Z^{-}} D_{0 \lambda^{\prime}}^{J\left(Z^{-}\right) *}\left(0, \theta_{Z^{-}}, 0\right) D_{\lambda^{\prime} \xi}^{1 *}\left(\tilde{\varphi}, \tilde{\theta}_{\psi^{\prime}}, \alpha\right) \\
& =H_{\lambda^{\prime}}^{Z^{-}} d_{0 \lambda^{\prime}}^{J\left(Z^{-}\right)}\left(\theta_{Z^{-}}\right) e^{i \lambda^{\prime} \tilde{\varphi}} d_{\lambda^{\prime} \xi}^{1}\left(\tilde{\theta}_{\psi^{\prime}}\right) e^{i \xi \alpha},
\end{aligned}
$$

where $H_{\lambda^{\prime}}^{Z^{-}}$is the helicity amplitude and $\lambda^{\prime}$ is the helicity of the $\psi^{\prime}$. The amplitudes in Eq. (22) are related by parity conservation in the decay $Z^{-} \rightarrow \psi^{\prime} \pi^{-}$:

$$
H_{\lambda^{\prime}}^{Z^{-}}=-P\left(Z^{-}\right)(-1)^{J\left(Z^{-}\right)} H_{-\lambda^{\prime}}^{Z^{-}}
$$

Note that the amplitudes in Eq. (17) for $\lambda$ and $-\lambda$ are not related, because the $\psi^{\prime}$ is produced in the weak decay $B^{0} \rightarrow \psi^{\prime} K^{*}$.

\section{The signal density function}

Combining the amplitudes in Eqs. (17) and (22), one gets the signal density function for $B^{0} \rightarrow \psi^{\prime} K^{+} \pi^{-}$decays:

$$
S(\Phi)=\sum_{\xi=1,-1}\left|\sum_{K^{*}} \sum_{\lambda=-1,0,1} A_{\lambda \xi}^{K^{*}}+\sum_{\lambda^{\prime}=-1,0,1} A_{\lambda^{\prime} \xi}^{Z^{-}}\right|^{2}
$$

The lepton pair is produced in the electromagnetic decay $\psi^{\prime} \rightarrow \ell^{+} \ell^{-}$via a virtual photon; thus its helicity $\xi$ may be equal to 1 or -1 .

For the charge conjugate decay $\bar{B}^{0} \rightarrow \psi^{\prime} K^{-} \pi^{+}$, the particles in the definitions of the angular variables change to the corresponding antiparticles $\left(K^{+} \rightarrow K^{-}, \pi^{-} \rightarrow\right.$ $\pi^{+}, \ell^{+} \rightarrow \ell^{-}$and $\left.\ell^{-} \rightarrow \ell^{+}\right)$. If the parity transformation is applied, then the helicity angles do not change and the azimuthal angles change sign (because $\cos \tilde{\varphi} \rightarrow \cos \tilde{\varphi}$ and $\sin \tilde{\varphi} \rightarrow-\sin \tilde{\varphi})$. Thus, the signal density for the decay $\bar{B}^{0} \rightarrow \psi^{\prime} K^{-} \pi^{+}$is given by Eq. (24) with the opposite sign of the azimuthal angles $(\varphi \rightarrow-\varphi, \tilde{\varphi} \rightarrow-\tilde{\varphi}$ and $\alpha \rightarrow-\alpha)$. 